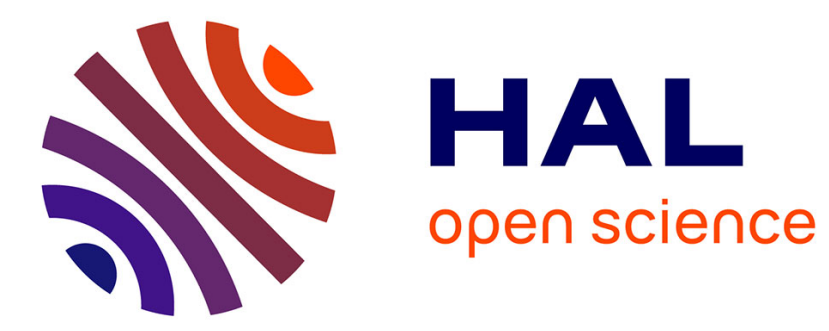

\title{
Geiger avalanche photodiodes as tentative light detectors for VHE gamma ray astronomy
}

Denis Pellion, K. Jradi, A. Le Padellec, Abdelali Rennane, F. Moutier, V. Borrel, Daniel Estève, C. Magenc, A. R. Bazer-Bachi

\section{- To cite this version:}

Denis Pellion, K. Jradi, A. Le Padellec, Abdelali Rennane, F. Moutier, et al.. Geiger avalanche photodiodes as tentative light detectors for VHE gamma ray astronomy. Experimental Astronomy, 2009, 27 (3), p. 187-195. 10.1007/s10686-009-9182-5 . hal-00637323

\section{HAL Id: hal-00637323 \\ https://hal.science/hal-00637323}

Submitted on 7 Nov 2011

HAL is a multi-disciplinary open access archive for the deposit and dissemination of scientific research documents, whether they are published or not. The documents may come from teaching and research institutions in France or abroad, or from public or private research centers.
L'archive ouverte pluridisciplinaire HAL, est destinée au dépôt et à la diffusion de documents scientifiques de niveau recherche, publiés ou non, émanant des établissements d'enseignement et de recherche français ou étrangers, des laboratoires publics ou privés. 


\title{
Geiger avalanche photodiodes as tentative light detectors for VHE gamma ray astronomy
}

\author{
D. Pellion • K. Jradi • A. Le Padellec • \\ A. Rennane · F. Moutier • V. Borrel · D. Esteve • \\ C. Magenc • A. R. Bazer-Bachi
}

Received: 11 May 2009 / Accepted: 23 November 2009

(C) Springer Science + Business Media B.V. 2009

\begin{abstract}
Due to its sensitivity and speed, the detector still widely used in Cerenkov astrophysics experiments remains the PhotoMultiplier Tube (PMT). For instance, recent pathbreaking experiments in Very High Energy astrophysics (VHE), such as MAGIC and HESS, have used mainstream PMT technology (Aharonian et al, Astron Astrophys 492(1):L25-L28, 2008). Moreover the Cerenkov Telescope Array (CTA) which is now in its design phase, is also planed to be based on PMT's. However, there are some disadvantages to the PMT technology: the rather poor quantum efficiency, the use of high voltages, the high cost when used in large number in a matrix arrangement and the large weight. Hence, we have investigated the possibility to design future Cerenkov telescopes based on solid state technology, specifically Geiger avalanche photodiodes. In a preliminary development
\end{abstract}

D. Pellion · K. Jradi · A. Le Padellec $(\bowtie)$ A A. Rennane · F. Moutier · V. Borrel ·

C. Magenc . A. R. Bazer-Bachi

Université de Toulouse; UPS; CESR, 9 avenue du Colonel Roche,

31028 Toulouse Cedex 9, France

e-mail: arnaud.lepadellec@cesr.fr

D. Pellion · K. Jradi · A. Le Padellec · A. Rennane · F. Moutier · V. Borrel ·

C. Magenc $\cdot$ A. R. Bazer-Bachi

CNRS, UMR5187, 31028 Toulouse, France

D. Esteve

Université de Toulouse; UPS; LAAS; 7 avenue du Colonel Roche, 31077 Toulouse, France

D. Esteve

CNRS, UPR8000, 31077 Toulouse, France 
test, we placed HAMAMATSU avalanche photodiodes at the focal plane of a $60 \mathrm{~cm}$ diameter telescope at the Pic du Midi in the French Pyrénées, in order to record incident cosmic rays. In this paper, we describe not only the experimental setup but we also put special emphasis to the reduction of the semi-conductor noise. We also show first data that were recorded during two runs in the fall of 2006, and conclude by the presentation of the design of an "integrated, low-cost solid state photodiode arrangement" which might be an alternative to PMT's for future VHE telescopes.

Keywords $\mathrm{SiPM} \cdot$ Cerenkov astronomy

\section{Introduction}

Early VHE gamma-ray astrophysics can be dated back to the early 80 's, when the Whipple experiment showed the existence of gamma rays at $\mathrm{TeV}$ energies [1]. Later on, a handful of sources were found by means of first generation telescopes such as AsGat, CAT or Celeste [2-4]. At the time, two different types of techniques were used, namely imaging-CAT_or sampling of the wave front-Celeste-. The present generation, with MAGIC [5] and HESS [6], combines both, and therefore allows stereoscopic imaging.

A modern Cerenkov telescope makes use of a large mirror to concentrate light on a detector focal plane. There, photodetectors convert the short Cerenkov light signal into an electrical pulse, further digitized by fast ADCs. Due to photons arising from star light and to unavoidable noise induced within any detector, there is a sizeable spurious counting rate at the level of an individual detector, whatever the quality of the local sky. This noise can be rejected by means of a carefully designed triggering system. Once filtered from spurious signals, the events of interest are analysed to discriminate between photon and hadron induced showers. The quality of this discrimination depends largely on the quality of the Monte-Carlo simulations used during the design phase.

Until now, the photodetectors used were photomultiplier tubes (PMT's). We are investigating in the present work the possibility to use solid state devices. We will describe below the experimental set-up we have used to measure high energy cosmic ray showers. Finally, the results will be presented, to conclude with perspectives for further developments.

\section{Towards a solid-state Cerenkov telescope}

Photomultiplier tubes (PMTs) have been used-so far-in all Cerenkov telescopes. The main reason is that they have the appropriate sensitivity and speed, allowing the detection of the single photo-electron with ns response. A single PMT is relatively cheap, and its technology has been well mastered for years. This is the reason why MAGIC II and HESS-II, as well as the future CTA, are 
now being built or designed with this PMT technology in mind. However there are nowadays new photodetectors on the market that are based on solid state devices.

Avalanche photodiodes have been used for over 20 years, but their gains, in the $10^{2}$ to $10^{3}$ range, have been insufficient to be an alternative to PMT. Nonetheless, a special mode for running APD's makes them serious challengers for PMT's: the so-called Geiger mode. When used cautiously at about $10 \%$ above its breakdown voltage, an APD can have a gain as high as $10^{6}$ to $10^{7}$.

About 5 years ago, substantial breakthroughts were achieved within the Geiger regime which are now useful in Cerenkov studies. The presently reported experiment is a preliminary setup of a basic Cerenkov detector for which we have purchased commercially available APD's. We installed five of them at the focal plane of a small telescope. The question was then: can such a simple setup detect VHE cosmic rays with a good confidence level?

\section{Experimental set-up and data reduction}

A Geiger APD, properly biased, displays a very high gain. It requires only two resistors to operate, one of $100 \mathrm{k} \Omega$ in order to control the current, the other one of $50 \Omega$ in order to collect the signal. Because of the relatively high bias voltage, the diode is ready to fire at any moment, and this can happen in two cases:

- a photon has fallen onto the surface, and the avalanche has been initiated very rapidly, in a timescale of a few picoseconds. It delivers a voltage pulse across the $50 \Omega$ resistor. Hence, charges flow very rapidly and the voltage falls below its breakdown value [7]. Then, in a matter of tens of ns, the system recovers depending upon the capacitance of the diode, and is again available for a new avalanche. The quenching can be passive or active; it is faster in the latter case, though this requires command transistors.

- a thermally generated electron initiates the avalanche, the most frequent case at room temperature. The thermal noise counting rate decreases by a factor of two for a decrease in temperature of $8^{\circ} \mathrm{C}$ [8]. This means that if we want to have reproductible data, the temperature has to be well known and regulated, i.e. by means of Peltier components.

It is necessary to reduce the dark count rate, dominated by thermal events. A commonly used method in VHE astrophysics is the triggering on a minimum of threefold coincidences. In doing so, one can ascertain that an event was initiated by a Cerenkov isochronous conical wavefront. Such a coincidence scheme is illustrated in Fig. 1 for twofold events and is a standard scheme in nuclear physics experiments.

We purchased a few Hamamatsu S9251 APDs, and checked their breakdown voltages and dark currents. The characteristics of these photodiodes are 
Fig. 1 Twofold coincidence scheme

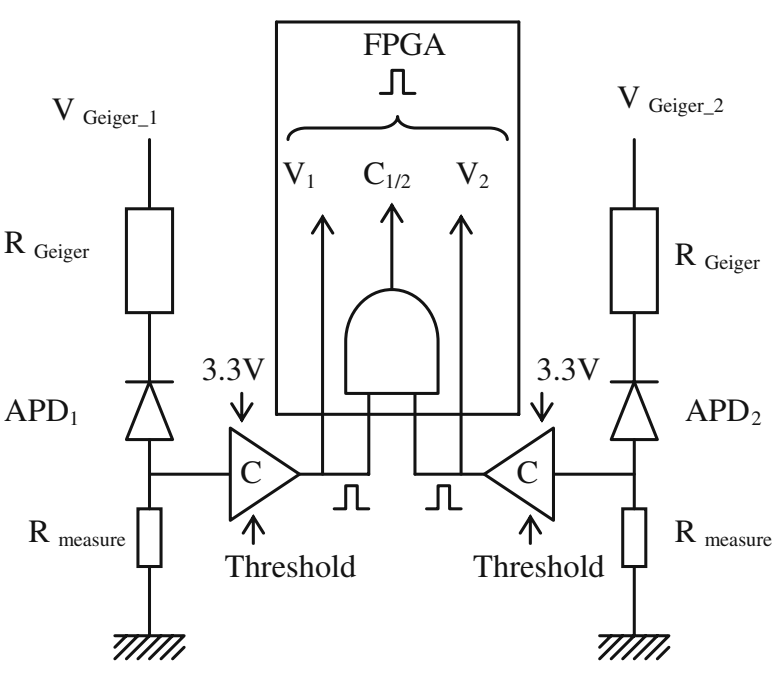

summarized in Table 1 . We made a selection of five similar diodes with a dark count rate of $50 \mathrm{kHz}$ at $20^{\circ} \mathrm{C}$ (temperature range investigated $-20^{\circ} \mathrm{C}$ to $30^{\circ} \mathrm{C}$ ) when they are operated $20 \mathrm{~V}$ above the breakdown voltage. The latter is defined by the appearance of counts at the output.

In addition to the dark count rate, one has to consider the additional night sky backgroud that accounts for $100 \mathrm{kHz}$. This is obtained while considering the $1.8 \%$ active surface of our detector, with an overall sky background of $100 \mathrm{MHz}$ per $\mathrm{m}^{2}$ and a $0.28 \mathrm{~m}^{2}$ collecting surface of our T60 télescope.

Since the cosmic ray count rate is expected to be of a few $\mathrm{Hz}$, most of the rate recorded by the diodes is due to the unavoidable thermal and nightsky background contributions. To eliminate this spurious signal, we used the five aforementionned diodes in a coincidence detection scheme. The diode output signal goes through a S\&H circuit that has a level control to adjust the threshold as can be seen in the example displayed in Fig. 1. The output of the comparator circuit has a width of $40 \mathrm{~ns}$. The Field-Programmable Gate Array (FPGA) counter allows one to register individual counting rates as well as any combinations of two, three, four and fivefold coincidences.

For illustration purposes, Fig. 2 displays a diode noise distribution as measured with the scheme in Fig. 1. It represents the time interval distribution between two subsequent pulses from a given photodiode. No signal is observed within the time interval 0.01 to $1 \mu$ s this being due not only to the photodiode placed in the dark at $20^{\circ} \mathrm{C}$ and polarized at $300 \mathrm{~V}$ (contribution of about $40 \mathrm{~ns}$ ), but also to extra resistors placed further downstream in the electronic circuit.

Table 1 Characteristics of the Hamamatsu photodiodes

\begin{tabular}{ll}
\hline Dark count rate & $50 \mathrm{kHz}$ \\
Vbr & $280 \mathrm{~V}$ \\
VGeiger & $300 \mathrm{~V}$ \\
Active diameter $\varnothing$ & $200 \mu \mathrm{m}$ \\
\hline
\end{tabular}


Fig. 2 Experimental noise distribution in a logarithmic scale

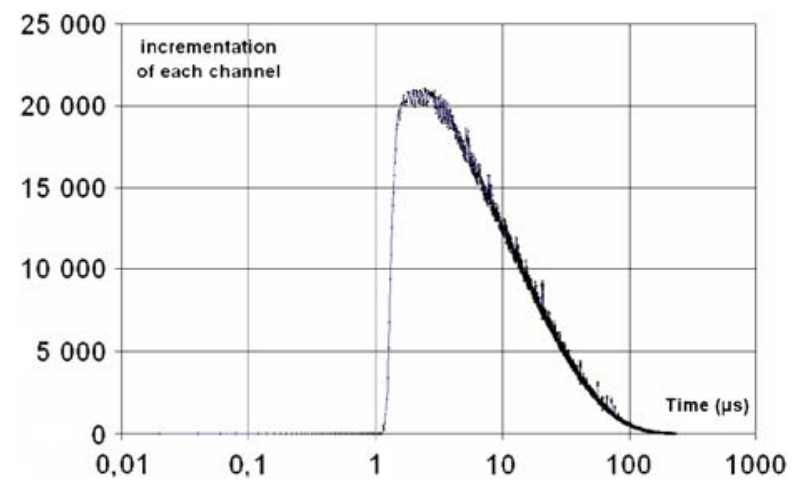

In order to model and evaluate the noise in the various coincidence configurations, one needs - first of all - to consider the noise as 100\% random. One has also to simplify the shape of the noise distribution, considering a maximal time separation between two adjacent pulses of $40 \mu \mathrm{s}$, thus twice that given by the average frequency of $50 \mathrm{kHz}$. The minimal time separation is thus equal to zero.

The probability $P_{A 1}$ to have the binary signal ' 1 ' is given by

$$
P_{A 1}=\frac{f_{g 1} L_{i}}{f_{g 1} L_{i}+2}
$$

with $f_{g}=50 \mathrm{kHz}$ the frequency in the Geiger mode and $L_{i}=40 \mathrm{~ns}$ the width of the pulse. With three identical photodiodes, the probability to have the three signals ' 1 ' (threefold coincidence) is therefore given by

$$
P_{A 3}=\left(\frac{f_{g 1} L_{i}}{f_{g 1} L_{i}+2}\right)^{3} .
$$

In order to extract the frequency for threefold coincidences, one needs first to calculate the coincidence width

$$
L_{i c 3}=\frac{\int_{0}^{\frac{L i}{2}} t d t+\left(L_{i}-\frac{L_{i}}{2}\right) *\left(L_{i}-\frac{L_{i}}{2}\right)+\int_{0}^{\frac{L i}{2}} t d t}{\frac{L_{i}}{2}+\frac{L_{i}}{2}+\frac{L_{i}}{2}}=\frac{L_{i}}{3} .
$$

Numerically, this equals 13.3 ns. One gets then the threefold coincidence probability

$$
P_{C 3}=\frac{L_{i c 3}}{L_{i c 3}+x}=\frac{L_{i}}{L_{i}+3 x}
$$


Table 2 Comparison computed/experimental noise frequencies (two, three, four, and fivefold coincidences)

\begin{tabular}{llll}
\hline $\begin{array}{l}\text { Number of } \\
\text { photodiodes }\end{array}$ & $\begin{array}{l}\text { Measured electronic } \\
\text { coincidence noise }\end{array}$ & $\begin{array}{l}\text { Calculated electronic } \\
\text { coincidence noise }\end{array}$ & $\begin{array}{l}\text { Calculated background }+ \\
\text { electronic coincidences noise }\end{array}$ \\
\hline 2 & $73 \mathrm{~Hz}$ & $74 \mathrm{~Hz}$ & $850 \mathrm{~Hz}$ \\
3 & $0.14 \mathrm{~Hz}$ & $0.15 \mathrm{~Hz}$ & $1 \mathrm{~Hz}$ \\
4 & $0.09 \mathrm{mHz}$ & $0.1 \mathrm{mHz}$ & $0.7 \mathrm{mHz}$ \\
5 & Not measurable & $0.0001 \mathrm{mHz}$ & $0.0002 \mathrm{mHz}$ \\
\hline
\end{tabular}

with $L_{i c 3}+x$ being the maximal time between two adjacent threefold coincidence events. The frequency for threefold coincidences is obtained through the equality $P_{A 3}=P_{C 3}$ which in terms gives the value for

$$
x=\frac{2\left(3 f_{g 1}^{2} L_{i}^{2}+6 f_{g 1} L_{i}+4\right)}{3 f_{g 1}^{3} L_{i}^{2}} .
$$

Subsequently, the period $T_{c 3}=\frac{\left(L_{i c}+x\right)}{2}$ gives the frequency

$$
f_{c 3}=\frac{6 f_{g 1}^{3} L_{i}^{2}}{f_{g 1}^{3} L_{i}^{3}+6 f_{g 1}^{2} L_{i}^{2}+12 f_{g 1} L_{i}+8} .
$$

The model calculations give very satisfactory outputs as they compare to experimental findings. These are summarized in Table 2, for two to fivefold coincidences.

\section{Fivefold coincidence measurements of Cerenkov flashes}

Convinced that a fivefold scheme would allow us to completely get rid of spurious noise, we performed measurements in december 2006, at the 2,877 m altitude of Pic du Midi de Bigorre-French Pyrénées, at an average temperature of $6^{\circ} \mathrm{C}$. We placed our package of five Hamamatsu photodiodes working in coincidence at the focal plane of the $60 \mathrm{~cm}$ diameter telescope. We measured the number of Cerenkov flashes as a function of the zenith angle (counted with respect to the local vertical direction). Our results are presented in Fig. 3.

Fig. 3 Coincidence frequency as a function of the zenith angle, (1) Normalized coincidence frequency as function of the zenith angle $\theta_{\mathrm{v}},(2) \mathrm{a} \cos ^{2} \theta_{\mathrm{v}}$ fit

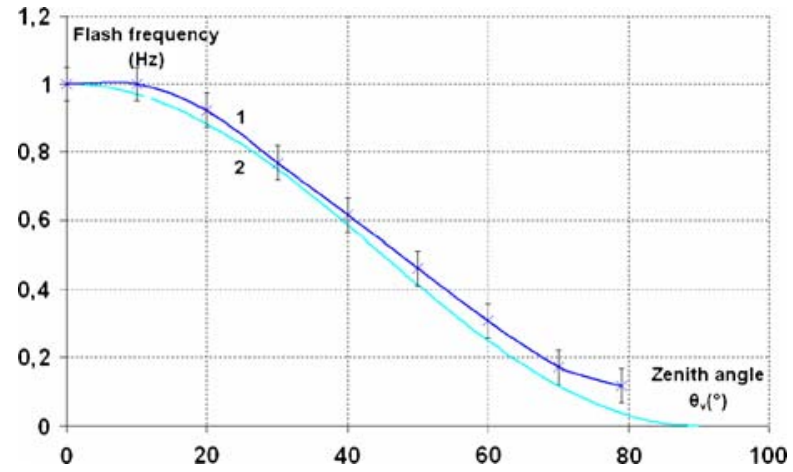


Curve $\mathrm{n}^{\circ} 1$ represents the normalized coincidence frequency as a function of the zenith angle $\theta_{\mathrm{v}}$ whereas curve $\mathrm{n}^{\circ} 2$ is the $\cos ^{2} \theta_{\mathrm{v}}$ dependence expected for low energy cosmic rays at ground level (see for instance Refs $[9,10]$ ). The reason for the latter dependence is that the spectrum of cosmic rays is dominated by surviving muons, and that the atmospheric absorption follows the thickness of air crossed, thus increasing with the zenith angle. As can be seen, both curves match reasonably within the experimental uncertainties on the frequency and the absolute determination of the zenith angle. Therefore, we conclude that our data are only due to incident cosmic ray showers generating Cerenkov light wavefronts, with a very weak spurious frequency of $2 \times 10^{-7} \mathrm{~Hz}$.

\section{Conclusion and perspectives}

In this work, we have presented results of an experiment conducted 3 years ago, with the objective to use solid state photodetectors for future atmospheric Cerenkov detectors. Thanks to the use of coincidence circuits to reject spurious noise, the results are in agreement with the expected variations of absorption in the atmosphere.

We are now working on the construction of a modest telescope (4 $\mathrm{m}$ in diameter) which, we hope, can appear as an alternative to all-PMTs ones. One important argument would be the low cost of such a telescope compared to usual ones.

To conduct the work presented here, we used Hamamatsu APD's that were available at the time. Today, technology for photodetectors has evolved towards the so-called Silicon Photomultiplier (SiPM). arrangment [11]. A SiPM consists in the association of several diodes in parallel, i.e. up to several thousands. As displayed in Fig. 4, it yields a single output signal.

Fig. 4 A SiPM arrangment

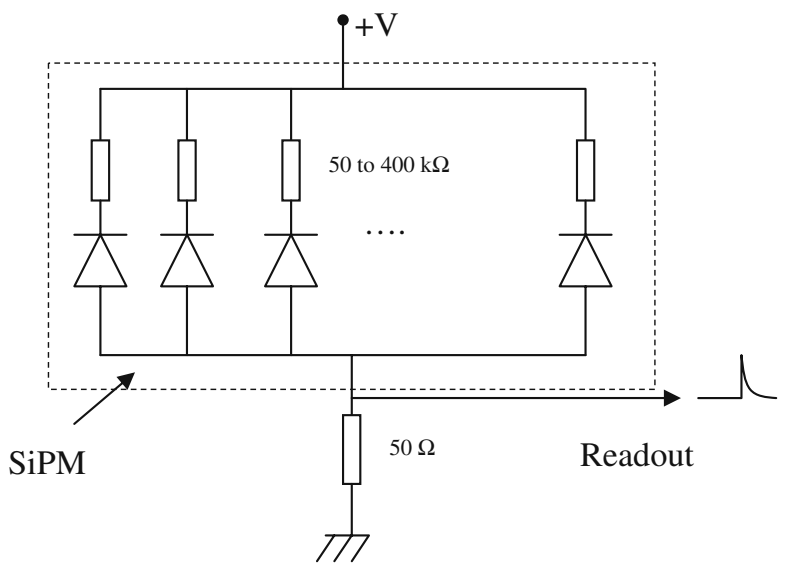


There are today several suppliers for SiPM: SensL, CPTA / Photonique, FBK-irst, Hamamatsu Photonics, MEPhI/Pulsar, RMD, SensL, ST-Microelectronics, Zecotek ..., and developments are conducted in a dozen laboratories worldwide. A typical SiPM has an area of $9 \mathrm{~mm}^{2}$. Our future plans concern measurements of the Cerenkov light that arises from $\gamma$ ray objects using $\mathrm{SiPM}$, that is to say we wish to build a 'SiPM Cerenkov telescope'. To do so, we have recently designed and manufactured a number of APD and SiPM photodetectors working in the Geiger photon counter mode, using our locally designed technology (associating CESR and LAAS laboratories). In the very near future, we plan to test them in the framework of a devoted 'Cherpic project', once again at the pic du Midi-french Pyrénées. Our prototype telescope will include all available technologies (microlenses, ASIC, integrated arrays of SiPM, a 512 pixels imager, ...). We plan to look at the Crab nebula source during the 2009-2010 winter season. Lastly, we should point out that some communications from other groups are starting to appear in the litterature on this very subject which are more or less at the same research stage $[12,13]$.

Acknowledgements The authors would like to thank Prof Adam Walters for valuable comments on the manuscript. This work was supported by Region Midi-Pyrénées and Observatoire Midi-Pyrénées (France).

\section{References}

1. Weekes, T.C., Cawlley, M.F., Fegan, D.J., Gibbs, K.G., Hillas, A.M., Kwok, P.W., Lamb, R.C., Lewis, D.A., Macomb, D., Porter, N.A., Reynolds, P.T., Vacanti, G.: Observation of TEV gamma-rays from the Crab-Nebula using the atmospheric Cerenkov imaging technique. Astrophys. J. 342(1), 379-395 (1989)

2. Goret, P., Palfrey, T., Tabary, A., Vacanti, G., Bazer-Bachi, R.: Observations of TEV gammarays from the Crab-Nebula. Astron. Astrophys. 270(1-2), 401-406 (1993)

3. Djannati-Atai, A., Piron, F., Barrau, A., Iacoucci, L., Punch, M., Tavernet, J.-P., Bazer-Bachi, R., Cabot, H., Chounet, L.M., Debiais, G., Degrange, B., Dezalay, J.-P., Dumora, D., Espigat, P., Fabre, B., Fleury, P., Fontaine, G., Ghesquiere, C., Goret, P., Gouiffes, C., Grenier, I.A., Le Bohec, S., Malet, I., Meynadier, C., Mohanty, G., Nuss, E., Pare, E., Quebert, J., Ragan, K., Renault, C., Rivoal, M., Rob, L., Schahmaneche, K., Smith, D.A.: Very high energy gamma-ray spectral properties of Mkn 501 from CAT Cerenkov telescope observations in 1997. Astron. Astrophys. 350(1), 17-24 (1999)

4. de Naurois, M., Holder, J., Bazer-Bachi, R., Begeret, H., Bruel, P., Cordier, A., Debiais, G., Dezalay, J.-P., Dumora, D., Durand, E., Eschstruth, P., Espigat, P., Fabre, B., Fleury, P., Herault, N., Hrabovsky, M., Incerti, S., Le Gallou, R., Munz, F., Musquere, A., Olive, J.-F., Pare, E., Quebert, J., Rannot, R.C., Reposeur, T., Rob, L., Roy, P., Sako, T., Schovanek, P., Smith, D.A., Snabre, P., Volte, A.: Measurement of the Crab flux above $60 \mathrm{GeV}$ with the Celeste Cerenkov telescope. Astrophys. J. 566(1), 343-357 (2002)

5. Albert, J., Aliu, E., Anderhub, H., Antoranz, P., Armada, A., Asensio, M., Baixeras, C., Barrio, J.A., Bartelt, M., Bartko, H., Bastieri, D., Bavikadi, R., Bednarek, W., Berger, K., Bigongiari, C., Biland, A., Bisesi, E., Bock, R.K., Bretz, T., Britvitch, I., Camara, M., Chilingarian, A., Ciprini, S., Coarasa, J.A., Commichau, S., Contreras, J.L., Cortina, J., Curtef, V., Danielyan, V., Dazzi, F., De Angelis, A., De Los Reyes, R., De Lotto, B., Domingo-Santamaria, E., Dorner, D., Doro, M., Errando, M., Fagiolini, M., Ferenc, D., Fernandez, E., Firpo, R., Flix, J., Fonseca, M.V., Font, L., Galante, N., Garczarczyk, M., Gaug, M., Giller, M., Goebel, F., Hakobyan, D., Hayashida, M., Hengstebeck, T., 
Hohne, D., Hose, J., Jacon, P., Kalekin, O., Kranich, D., Laille, A., Lenisa, T., Liebing, P., Lindfors, E., Longo, F., Lopez, J., Lopez, M., Lorenz, E., Lucarelli, F., Majumdar, P., Maneva, G., Mannheim, K., Mariotti, M., Martinez, M., Mase, K., Mazin, D., Meucci, M., Meyer, M., Miranda, J.M., Mirzoyan, R., Mizobuchi, S., Moralejo, A., Nilsson, K., Ona-Wilhelmi, E., Orduna, R., Otte, N., Oya, I., Paneque, D., Paoletti, R., Pasanen, M., Pascoli, D., Pauss, F., Pavel, N., Pegna, R., Persic, M., Peruzzo, L., Piccioli, A., Prandini, E., Rhode, W., Rico, J., Riegel, B., Rissi, M., Robert, A., Rugamer, S., Saggion, A., Sanchez, A., Sartori, P., Scalzotto, V., Schmitt, R., Schweizer, T., Shayduk, M., Shinozaki, K., Shore, S., Sidro, N., Sillanpaa, A., Sobczynska, D., Stamerra, A., Stark, L.S., Takalo, L., Temnikov, P., Tescaro, D., Teshima, M., Tonello, N., Torres, A., Torres, D.F., Turini, N., Vankov, H., Vardanyan, A., Vitale, V., Wagner, R.M., Wibig, T., Wittek, W., Zapatero, J.: Flux upper limit on gamma-ray emission by GRB 050713a from magic telescope observations. Astrophys. J. 641(1), L9-L12 (2006)

6. Aharonian, F., Akhperjanian, A.G., de Almeida, U., Bazer-Bachi, A.R., Becherini, Y., Behera, B., Benbow, W., Bernloehr, K., Boisson, C., Bochow, A., Borrel, V., Braun, I., Brion, E., Brucker, J., Brun, P., Buehler, R., Bulik, T., Buesching, I., Boutelier, T., Carrigan, S., Chadwick, P.M., Charbonnier, A., Chaves, R.C.G., Cheesebrough, A., Chounet, L.-M., Clapson, A.C., Coignet, G., Dalton, M., Degrange, B., Deil, C., Dickinson, H.J., Djannati-Atai, A., Domainko, W., Drury, L.O., Dubois, F., Dubus, G., Dyks, J., Dyrda, M., Egberts, K., Emmanoulopoulos, D., Espigat, P., Farnier, C., Feinstein, F., Fiasson, A., Foerster, A., Fontaine, G., Fuessling, M., Gabici, S., Gallant, Y.A., Gerard, L., Giebels, B., Glicenstein, J.F., Glueck, B., Goret, P., Hadjichristidis, C., Hauser, D., Hauser, M., Heinz, S., Heinzelmann, G., Henri, G., Hermann, G., Hinton, J.A., Hoffmann, A., Hofmann, W., Holleran, M., Hoppe, S., Horns, D., Jacholkowska, A., de Jager, O.C., Jung, I., Katarzynski, K., Kaufmann, S., Kendziorra, E., Kerschhaggl, M., Khangulyan, D., Khelifi, B., Keogh, D., Komin, N., Kosack, K., Lamanna, G., Lenain, J.-P., Lohse, T., Marandon, V., Martin, J.M., Martineau-Huynh, O., Marcowith, A., Maurin, D., McComb, T.J.L., Medina, M.C., Moderski, R., Moulin, E., Naumann-Godo, M., de Naurois, M., Nedbal, D., Nekrassov, D., Niemiec, J., Nolan, S.J., Ohm, S., Olive, J.-F., Wilhelmi, E.D., Orford, K.J., Osborne J.L., Ostrowski, M., Panter, M., Arribas, M., Pedaletti, G., Pelletier, G., Petrucci, P.-O., Pita, S., Puehlhofer, G., Punch, M., Quirrenbach, A., Raubenheimer, B.C., Raue, M., Rayner, S.M., Renaud, M., Rieger, F., Ripken, J., Rob, L., Rosier-Lees, S., Rowell, G., Rudak, B., Rulten, C.B., Ruppel, J., Sahakian, V., Santangelo, A., Schlickeiser, R., Schoeck, F.M., Schroeder, R., Schwanke, U., Schwarzburg, S., Schwemmer, S., Shalchi, A., Skilton, J.L., Sol, H., Spangler, D., Stawarz, L., Steenkamp, R., Stegmann, C., Superina, G., Tam, P.H., Tavernet, J.-P., Terrier, R., Tibolla, O., van Eldik, C., Vasileiadis, G., Venter, C., Vialle, J.P., Vincent, P., Vivier, M., Voelk, H.J., Volpe, F., Wagner, S.J., Ward, M., Zdziarski, A.A., Zech, A.: Simultaneous HESS and Chandra observations of Sagitarius A(star) during an X-ray flare. Astron. Astrophys. 492(1), L25-L28 (2008)

7. Zappa, F., Tisa, S., Tosi, A., Cova, S.: Principles and features of single-photon avalanche diode arrays. Sens. Actuators A, Phys. 140, 103-112 (2007)

8. Jackson, J.C., Phelan, D., Morrison, A.P., Redfern, M., Mathewson, A.: Toward integrated single-photon-counting microarrays. Opt. Eng. 1(42), 112-118 (2003)

9. Bhat, P.N., Murthy, P.V.R.: Angular-distribution of low-energy Cosmic-Ray Muons Underground. J. Phys. G., Nucl. Phys. 4(3), 453-469 (1978)

10. Cosmic Rays, Gaisser, T.K., Stanev, T., (Bartol Research Inst, Univ. of Delaware), 2002, Rev. Sokolsky, P.V. (Univ. of Utah), R.E.Streitmatter, 2005

11. Buzhan, P., Dolgoshein, B., Filatov, L., Ilyin, A., Kantzerov, V., Kaplin, V., Karakash, A., Kayumov, F., Klemin, S., Popova, E., Smirnov, S.: Silicon photomultiplier and its possible applications. Nucl. Instrum. Methods Phys. Res. A 504, 48-52 (2003)

12. Bitossi, M., Cecchi, R., Paoletti, R., Pegna, R., Turini, N., Barcelò, M., Illa, J.M.: The data acquisition system of the MAGiC-II telescope. In: IDAACS 2007: Proceedings of the 4th IEEE Workshop on Intelligent Data Acquisition and Advanced Computing Systems: Technology And Applications, pp. 60-62 (2007)

13. Biland, A., Britvich, I., Lorenz, E., Otte, N., Pauss, F., Renker, D., Ritt, S., Roesner, U., Scheebeli, M.: First detection of air shower Cerenkov light by Geigermode-Avalanche Photodiodes. Nucl. Instrum. Methods Phys. Res. Sect. A, Accel. Spectrom. Detect. Assoc. Equip. 595(1), 165-168 (2008) 